Revue de droit comparé du travail et de la sécurité sociale

2 | 2020

La vie personnelle du salarié

\title{
Tamás Gyulavári, Emanuele Menegatti (eds), The Sources of Labour Law
}

Wolters Kluwer, 2020

Sylvaine Laulom

\section{OpenEdition}

Journals

Édition électronique

URL : https://journals.openedition.org/rdctss/1138

DOI : $10.4000 /$ rdctss. 1138

ISSN : 2262-9815

Éditeur

Centre de droit comparé du travail et de la sécurité sociale

Édition imprimée

Date de publication : 1 juin 2020

Pagination : 138-141

ISSN : 2117-4350

Référence électronique

Sylvaine Laulom, "Tamás Gyulavári, Emanuele Menegatti (eds), The Sources of Labour Law », Revue de droit comparé du travail et de la sécurité sociale [En ligne], 2 | 2020, mis en ligne le 01 novembre 2021, consulté le 11 novembre 2021. URL : http://journals.openedition.org/rdctss/1138; DOI : https:// doi.org/10.4000/rdctss. 1138

\section{(c) $109 \Theta$}

Revue de droit comparé du travail et de la sécurité sociale est mise à disposition selon les termes de la Licence Creative Commons Attribution - Pas d'Utilisation Commerciale - Pas de Modification 4.0 International. 


\section{TAmás GyulavÁRI, EmanUele MenegatTI (eds), THE SOURCES OF LABOUR LAW Wolters Kluwer, 2020 , 404 p., Alphen aan den Rijn}

Les ouvrages de droit du travail comparé restent, aujourd'hui encore, trop rares et c'est l'un des grands mérites de cet ouvrage que de proposer un voyage sur les 5 continents pour ausculter l'état des sources du droit du travail. L'ampleur de l'ouvrage doit être souligné tant par l'objet analysé que par le champ géographique couvert. Ce ne sont pas moins de 15 pays qui sont analysés (Australie, Brésil, Chine, Danemark, France, Allemagne, Hongrie, Italie, Pologne, Russie, Espagne, Suède, Afrique du Sud, Royaume-Uni et Etats Unis). Si les pays européens restent majoritaires, l'ouvrage permet une plongée dans des systèmes juridiques d'une très grande variété et il offre un matériel inégalé permettant d'établir des comparaisons.

La structure de l'ouvrage est classique mais reste indépassable des approches comparatives: une première partie est consacrée à des approches "horizontales " ou transversales et internationales, la deuxième partie regroupe les articles dédiés aux situations nationales, articles qui suivent une structure commune permettant d'identifier des parallèles et des objets de comparaisons.

L'ouvrage est fondé sur une prémisse : le droit du travail, édifice unique des sociétés modernes, est aujourd'hui en péril, sa légitimité est questionnée. Les politiques de flexibilisation et de «modernisation » menées depuis des années à l'échelle européenne et nationales ont attaqué le contenu des règ les de droit du travail, mais plus fondamentalement sa structure. C'est précisément cette structure, cette architecture en évolution des sources du droit du travail qui fait l'objet de cette recherche, en constitue l'apport essentiel et son originalité.

Cinq chapitres constituent la première partie « horizontale » de l'ouvrage et proposent une approche originale des sources du droit du travail. On aurait pu s'attendre à une analyse des différentes sources, par exemple les sources constitutionnelles, les sources conventionnelles, la question de la hiérarchie ou de l'articulation des différentes sources entre elles. Les éditeurs de l'ouvrage ont, à juste titre, privilégié une autre approche en choisissant de demander à cinq spécialistes reconnus de traiter de thèmes transnationaux et/ou comparistes. Après un chapitre introductif par les éditeurs de l'ouvrage, dressant une première comparaison des évolutions nationales (Chap. 1 : "Introduction : recent trends in the Hierarchy of Labour Law Sources "), Alan Neal, dans un deuxième chapitre ( « Recalling some of the historical roots for twenty-first century approaches to regulation of the world of work), propose une reconstruction historique des racines du droit du travail et la centralité première du contrat de travail. L'un des intérêts majeurs de l'analyse d'Alan Neal est sans contexte d'introduire le droit chinois dans l'analyse. Deux chapitres de cette partie (chapitre 4 rédigé par Joellen Riley Munton, "Judge-Made Law in the Common Law World : a Conservative Influence on the Transformation of Labour Law by Statute », 
et chapitre 5, rédigé par Martin Risak, «Filling the (Increasing) Gaps : the Role of Judges as Substitute Legislators in Labour Law in the Civil Law Countries ») analysent ensuite le rôle du juge en droit du travail, dans les systèmes de Common law et dans les systèmes de droit civil où l'on voit, qu'en dépit d'une conception fondamentalement différente du juge et de la jurisprudence, leur rôle dans le développement du droit du travail apparaît remarquablement similaire. Ils démontrent ainsi à quel point l'opposition classique en droit comparé entre les pays de Common law et les pays de droit civil doit être relativisé en droit du travail, les pays pouvant mieux être analysés au regard notamment du rôle dévolu à l'autonomie collective. Les chapitres 3 et 6 - respectivement rédigés par Edoardo Ales, "The regulatory function of collective agreements in the light of its relationship with statutory instruments and individual rights : a multilevel approach » et Emanuele Menegatti, "The impact of the European Union economic governance on the hierarchy of national labour law sources » - partagent une approche internationale et européenne. Edoardo Ales analyse la fonction normative des accords collectifs en droit international et européen (entendu ici largement et intégrant tant le droit de l'Union européenne que le droit du Conseil de l'Europe). Du côté de l'OIT, la fonction normative des conventions collectives, c'est-à-dire la reconnaissance qu'il existe d'autres sources que la loi pour réglementer les conditions de travail et que ces sources conventionnelles peuvent produire les mêmes effets que la loi et prévaloir sur le contrat de travail, a été reconnue et soutenue dès l'instauration de l'organisation internationale. Comme le montre Edoardo Ales, l'Union européenne a également reconnu et soutenu cette fonction normative et nombreux sont les textes européens, qu'il s'agisse du droit dérivé ou des Traités et des chartes, qui reconnaissent et organisent cette fonction normative, y compris dans sa capacité dérogatoire. C'est d'ailleurs tout le paradoxe car la gouvernance économique européenne, comme le montre Emanuele Menegatti, a fortement milité pour que les Etats membres œuvrent à une restructuration de leurs sources du droit du travail, conduisant à une remise en cause, d'une part, du rôle de la législation et, d'autre part et surtout, des systèmes centralisés de relations collectives et de conventions collectives. A travers l'exemple de trois pays (France, Italie et Portugal), Emanuele Menegatti montre que les Etats ont repris à leur compte les injonctions européennes et ont, pour certains, profondément modifié leur législation en privilégiant une négociation collective dérogatoire d'entreprise. Cette décentralisation désorganisée, soutenue par (ou exigée de) l'Union européenne peut conduire à une individualisation de la réglementation du travail, susceptible à terme de conduire à un déclin irréversible du droit du travail, remplacé par un régime général de liberté contractuelle. La conclusion de ce chapitre nuance néanmoins cette perspective en mettant l'accent sur les capacités de résistance des systèmes nationaux.

C'est d'ailleurs tout l'intérêt de la deuxième partie que de proposer une perspective mondiale des évolutions des sources du droit du travail, où est en jeu l'avenir même du droit du travail. Le champ géographique couvert par l'ouvrage permet de proposer une cartographie des transformations récentes des sources du droit et de leur articulation hiérarchique. La flexibilisation du droit du travail à l'œuvre depuis plusieurs années n'induit pas qu'une transformation du contenu des normes mais elle affecte plus fondamentalement encore les sources de droit du travail. Traditionnellement, le principe de faveur a permis une articulation des sources de droit du travail protectrice des droits des salariés. Cette architecture traditionnelle du droit du travail se fissure à tel point que l'édifice est aujourd'hui en péril. La lecture des 15 chapitre nationaux offre néanmoins une vision beaucoup plus nuancée et surtout diversifiée de ces évolutions. Dans cette perspective, l'un des apports majeurs des rapports nationaux est de montrer l'originalité de chaque système et surtout les forces de résistance à l'œuvre. 